Proceedings of the 2010 Winter Simulation Conference

B. Johansson, S. Jain, J. Montoya-Torres, J. Hugan, and E. Yücesan, eds.

\title{
INCORPORATING HEALTHCARE SYSTEMS IN PANDEMIC MODELS
}

\author{
Natalia E. Lizon \\ Dionne M. Aleman \\ Department of Mechanical and Industrial Engineering \\ University of Toronto
5 King's College Road, Toronto, ON M5S 3G8, CANADA \\ Brian Schwartz \\ Department of Family and Community Medicine \\ University of Toronto \\ 263 McCaul Street, Toronto, ON M5T 1W7, CANADA
}

\begin{abstract}
There are several models used to predict the spread of disease in a pandemic, but few, if any, incorporate the effect of healthcare systems in preventing propagation of the disease. In areas where healthcare is easily available to the general public (specifically, countries with universal healthcare), the ability of infected individuals to receive rapid treatment should impact disease spread. Additionally, the presence of a pandemic will result in an increased load on the healthcare system as infected individuals seek medical attention at hospitals and from their family doctors. We modify an existing non-homogeneous, agent-based simulation pandemic disease spread model to incorporate a public healthcare system in a pandemic influenza simulation on the Greater Toronto Area, Ontario, Canada. Results show that healthcare availability significantly significantly increases disease spread due to increased contacts within the population. We also find that the creation of flu centers decreases flu-related deaths and decreases hospital admissions.
\end{abstract}

\section{INTRODUCTION}

In 2002-2003, a global outbreak of Severe Acute Respiratory Syndrome (SARS) resulted in over 8,000 infections and almost 800 deaths. This outbreak illustrated the need for not only pandemic planning, but also for accurate pandemic modeling. Realistic models for predicting the spread of disease can provide evidence as to the effects of mitigation strategies in lessening the spread of disease. For example, the effect of vaccinating individuals in certain age groups can be estimated in advance and compared to other possible strategies.

In this work, we are specifically interested in the effect of healthcare availability on disease spread, and the effect of disease spread on healthcare resources. We are additionally focused on the pilot region of the Greater Toronto Area (GTA), Ontario, Canada. The GTA consists of approximately 4.99 million people living in a largely urban environment. The presence of universal healthcare and wide usage of public transportation make this region difficult to model for most existing disease spread approaches.

The majority of pandemic disease spread models in the literature rely on simplifying measures that make incorporating healthcare systems complicated or even impossible. Homogeneous mixing models, the most common approach to disease spread modeling, assume that all infected individuals are assumed to transmit the disease to the same number of people (that number is called the basic reproduction number, $R_{0}$ ). This simplification has been shown to lead to inconsistent and inaccurate predictions of disease spread (Meyers et al. 2005, Newman 2002). Additionally, the uniformity of each individual of the population make it difficult to account for individualistic attributes such as use of public transportation or inclination to visit a family doctor once infected.

Although more realistic non-homogeneous (heterogeneous) mixing models exist (Meyers et al. 2005, Valle et al. 2007, Larson 2007), it is still difficult to address individualistic attributes. Instead, we employ a non-homogeneous agent-based simulation model (Aleman et al. 2009a, Aleman et al. 2009b) that allows for each individual in the population to be unique, including demographic information and behavioral patterns. The individual characteristics will allow us to simulate not only the number of infections and deaths caused by the disease, but also the impact on regional healthcare facilities. 
We examine the number of visits to hospital emergency departments (EDs) and general practitioners (GPs, also called family doctors) experienced during the pandemic. We compare these values to a situation where the government creates flu assessment, treatment and referral centers throughout the GTA ("flu centers") to test the effectiveness of this mitigation strategy. Furthermore, our results show that just the availability of healthcare significantly impacts the spread of disease, and so models without such considerations may not be applicable to areas where there is universal healthcare.

\section{NON-HOMOGENEOUS AGENT-BASED SIMULATION MODEL}

We employ the non-homogeneous agent-based simulation model presented in the works of Aleman et al. (2009a), Aleman et al. (2009b). This model considers the three factors that allow for disease transmission: ambient environment, the infectivity of the affected individual, and the vulnerability of the infected individual. This model calculates the probability of infection of any member of the population using Markov chain theory as shown in Figure 1. In the Markov chain, each individual is classified as susceptible (S), infected (I) or removed ( $\mathrm{R})$. This type of classification is called an S-I-R model.

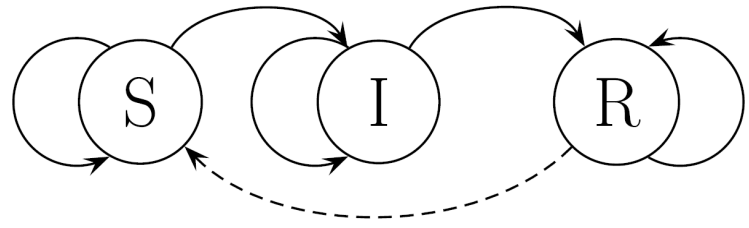

Figure 1: Markov chain of transition probabilities in the S-I-R model

Once an individual is infected (i.e., transitions from state $S$ to state I), the length of time that s/he stays in state I can be determined by factors including age, gender, ethnicity, co-morbidities, or any other available information deemed pertinent in predicting duration of infection in an individual. The probability of that patient transitioning to state $\mathrm{R}$ before the calculated number of days that person remains infected (that is, the person dies), can also be similarly determined. State $\mathrm{R}$ is treated as an absorbing state for the pandemic influenza strain simulated in this work, but in the event of a rapidly mutating disease where recovered individuals do not retain immunity, an individual who transitions into state $\mathrm{R}$ can be forced to transition back to state $\mathrm{S}$. This possibility is represented by the dotted arc connecting states $\mathrm{R}$ and $\mathrm{S}$.

In any time period, two individuals have some probability of coming into contact with each other based on their household membership, public transportation usage or healthcare usage (e.g., patient-nurse contact), as well as casual contact such as shaking hands or close (within three feet) conversation. If an individual is infected, then all the individuals with which that person has contact have a non-zero probability of becoming infected in the next time period. This probability is transition probability from state $\mathrm{S}$ to state I and is calculated by

$$
\operatorname{Pr}_{j}^{n}(\mathrm{~S}, \mathrm{I})=\underset{i \in \mathscr{I}}{ }\left(t_{i j}^{n} b_{i j}^{n}+c_{i j}^{n}\right) \quad \forall j \in \mathscr{S}
$$

where $\mathscr{S} \subseteq \mathscr{N}$ is the set of susceptible individuals in population $\mathscr{N} ; \mathscr{I} \subseteq \mathscr{N}$ is the set of infected individuals in population $\mathscr{N} ; t_{i j}^{n}$ is the time of contact between person $i$ and person $j$ in time period $n$; and $b_{i j}^{n}$ is the probability of disease transmission from person $i$ to person $j$ per unit time in time period $n$. Indirect contact between two individuals is controlled by the value $c_{i j}^{n}$, which is independent of the amount of time the two individuals are in the same place. In fact, infected individual $i$ and healthy individual $j$ may not ever be at the same place at the same time; but if person $i$ was in the same location as that person $j$ later, person $i$ 's microorganisms could still be transmitted to person $j$ via contact with fomites or surfaces in the environment.

The specifics of the population generation, transmission probabilities and model design are given in (Aleman et al. 2009a, Aleman et al. 2009b), and are not further discussed here.

\subsection{Incorporation of healthcare facilities}

The extensions made to this model are to allow for individuals to seek medical attention once they become ill. Medical attention is delivered by family doctors or hospital emergency departments near the infected individual's home. Assignment to a medical facility is based on the regional boundaries of Ontario's Local Health Integration Networks (LHINs), not-for-profit organizations that oversee the administration of Ontario's 
Lizon, Aleman and Schwartz

\begin{tabular}{ccccc}
\hline & \multicolumn{5}{c}{ Age group of infectious person } \\
Age group of susceptible nurse & $0-4$ & $5-18$ & $19-64$ & $64+$ \\
\hline $19-64$ & 1,10 & 1,10 & 1,10 & 1,10 \\
\hline
\end{tabular}

Table 1: Number of contacts and time of each contact with between infected nurses and patients

\begin{tabular}{lc}
\hline Contact & Probability \\
\hline Infected nurse with susceptible patient & 0.250000 \\
Susceptible nurse with infected patient & 0.250000 \\
Infected patient with susceptible patient & 0.000219 \\
\hline
\end{tabular}

Table 2: Estimated contact probabilities

healthcare budget. There are 14 LHINs that encompass the province of Ontario, and five of them address the GTA.

The model assigns individuals to households located in census tracts according to census data, and each census tract is serviced by a specific LHIN. The probability of individual being in a particular LHIN's jurisdiction is given in (Central West LHIN 2004, Mississauga Halton LHIN 2004, Toronto Central LHIN 2004, Central LHIN 2008, Central East LHIN 2009). Each of these five LHINs covering the GTA contains between two and nine hospitals (Ontario Hospital Association 2007), and an individual in a particular LHIN seeking medical attention will uniformly randomly visit one of the hospitals within the LHIN.

We can also use the LHINs to calculate the number of ED physicians, primary care physicians, ED nurses and intensive care unit (ICU) nurses per LHIN (Ontario Physician Human Resources Data Centre 2008, National Physician Survey 2007), as well as the probability that an individual in a LHIN is one of these clinician types. An individual who is an ED Nurse, an ICU nurse or an ED physician is assigned to a hospital within his/her home LHIN. The probability of working at each hospital for a given LHIN is uniformly distributed for each hospital.

Each individual is also assigned to be either an acute or non-acute patient, based on a 3.29\% annual probability of visiting an emergency department in Ontario (Institute for Clinical Evaluative Sciences 2000). These acute patients visit the ED for reasons other than pandemic influenza. The probability of an individual visiting the ED due to pandemic influenza was assumed to be $10 \%$, while a visit to a GPs was estimated to be 40\% (Ontario Health Plan for an Influenza Pandemic 2008). The remaining 50\% of the population did not receive care, and we estimated $15 \%$ stayed home while infected, and 35\% continued about their usual daily business. These values were informed by expert opinion and intuition, but can be adjusted as necessary to reflect the population's true behavior should more data become available.

Once an individual visits a hospital, there is a probability of disease transmission from the infected patient to nurses. Contact times and durations based on expert opinion from infectious disease clinicians at Mount Sinai Hospital in Toronto, Ontario are given in Table 1.

We additionally incorporate the contact probabilities shown in Table 2 . The $25 \%$ probabilities given for the two types of nurse contact with patients arise from the simplifying assumption that there are four nurses working on an ED shift at any time. The probability of an infected patient having contact with a susceptible patient is the probability that a non-infected individual will visit the hospital during a 60-day simulation for non-pandemic related reasons (3.29\% chance) divided by 60 to indicate probability of an ED visit on a particular day; this number is further multiplied by $40 \%$ to represent a $40 \%$ chance that two individuals will be within the requisite two-meter radius proximity of each other for disease transmission to occur (Low et al. 2007).

Patients who are admitted to a hospital in-patient bed or to the ICU are assumed to remain in the hospital and then stay home throughout the remainder of the model. The length of stay expected for an influenza patient in an acute bed is seven days (Toronto Academic Health Sciences Network 2006), and for an influenza patient in an intensive care bed is ten days (Centers for Disease Control and Prevention 2005, Toronto Academic Health Sciences Network 2006). 
Lizon, Aleman and Schwartz.

\begin{tabular}{lccc}
\hline Behavior & No healthcare & Healthcare & Flu centers \\
\hline Visit hospital ED & 0 & 0.10 & 0.05 \\
Visit primary care doctor & 0 & 0.40 & 0.70 \\
Stay at home & 0.15 & 0.15 & 0.10 \\
Continue with work/school & 0.85 & 0.35 & 0.15 \\
\hline
\end{tabular}

Table 3: Probability of a patient behaviour once infected

\begin{tabular}{lccc|ccc}
\hline & \multicolumn{3}{c|}{ Infections } & \multicolumn{3}{c}{ Deaths } \\
& No healthcare & Healthcare & Flu centers & No healthcare & Healthcare & Flu centers \\
\hline Average & 25,354 & $1,502,984$ & $1,231,828$ & 1,383 & 54,448 & 34,047 \\
St. Dev. & 510 & 246,886 & 469,208 & 44 & 8,951 & 12,978 \\
Minimum & 22,873 & 14 & 14 & 1,220 & 0 & 0 \\
Maximum & 26,446 & $1,569,207$ & $1,454,763$ & 1,509 & 57,388 & 40,418 \\
\hline
\end{tabular}

Table 4: Statistical results of total number of infections and deaths

\subsection{Mitigation strategy assessment}

The mitigation strategy assessed in this work is the introduction of flu centers. It is thought that flu centers can reduce the load on hospitals while simultaneously lowering the number of infections and deaths. This model tests the effectiveness of establishing flu centers through the GTA based on the patient behavior assumptions in Table 3, where visiting a flu center is treated as equivalent to visiting a primary care physician.

The model is run both with and without flu centers to assess the relative effectiveness of employing flu centers. The resulting numbers of infected and dead individuals, as well as the load on the healthcare system, were compared to address the impact of flu centres on disease spread. Load on the healthcare system is assessed by collecting information on the number of acute patients, ICU patients and primary care patients seen in the simulation. We additionally keep track of the number of ED nurses, ICU nurses, ED doctors and primary care physicians who become infected during the outbreak.

We also compare the base disease spread model (no healthcare system) to our modified model that includes healthcare availability to examine the impact of healthcare on disease spread.

\section{RESULTS}

The model is written in $\mathrm{C}++$ and was run on a 32-node, 256-processor Beowulf high performance computing infrastructure to obtain 1,000 simulations of disease spread outcomes over the course of a 100-day outbreak. This number of iterations was chosen based on sensitivity analysis indicating that 1,000 simulations is sufficient to establish a stable distribution of results.

Compared to the original implementation of the disease spread model presented in (Aleman et al. 2009a, Aleman et al. 2009b), which did not include healthcare availability, the inclusion of the health system dramatically increased the spread of the pandemic outbreak due to additional opportunities for contact and disease transmission (Table 4). However, the number of deaths did not rise proportionally when healthcare was added into the model due to the assumption that individuals receiving medical attention would be only half as likely to die from the infection as individuals who do not seek professional care.

With flu centers, the number of infected ED nurses decreased by an average of $18.3 \%$, ICU nurses by $24 \%$ and ED doctors by $23 \%$. Somewhat surprisingly, the number of infections among primary care physicians also dropped by an average of $19 \%$ with the introduction of flu centers. As expected, the average reduction in influenza patients presenting at EDs was 59\%.

Figure 2 demonstrate the impact on disease spread made by the public health system in Ontario. These results show that with $99 \%$ confidence, the lack of inclusion of the health system underestimates the spread of disease. Although the number of infections between healthcare and and healthcare with flu centers did not differ in a statistically significant way, the number of deaths with flu centers was reduced in $97.8 \%$ of trials. However, in the $2.2 \%$ of simulations where the number of deaths with flu centers increased over not having 
flu centers, the difference in deaths was significant enough to result in only $80 \%$ overall confidence that flu centers reduce the number of deaths.
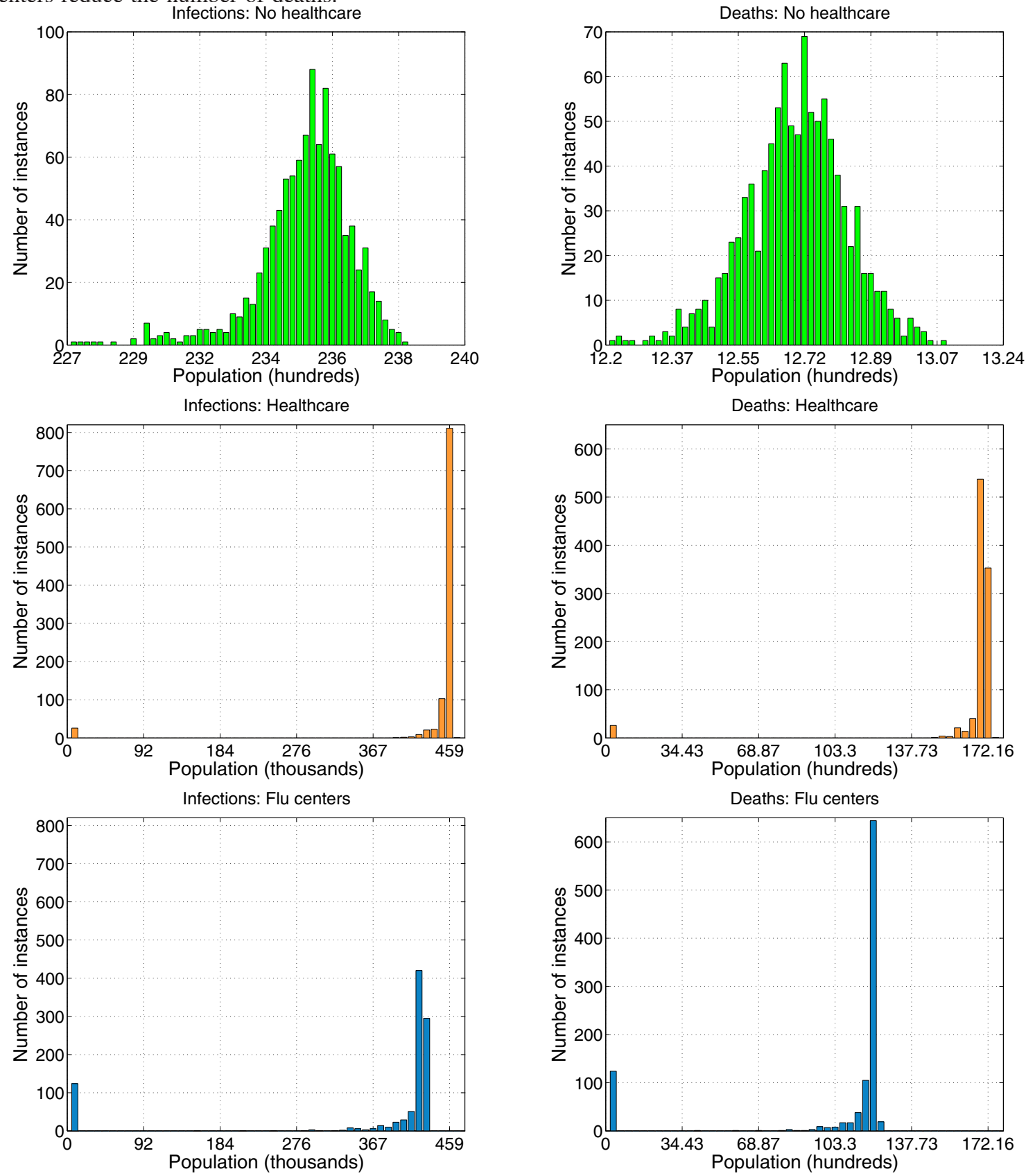

Figure 2: Population outcomes in terms of infections (left) and deaths (right). Top: No healthcare (shown with a different scale than the other scenarios). Middle: Healthcare without flu centers. Bottom: Healthcare with flu centers.

Statistical confidence was assessed using confidence intervals in the following manner. Because common random numbers were used across scenarios, we can examine the difference in all 1,000 outcomes from scenario $i$ to scenario $j$ and calculate the difference in, say, deaths, from scenario $i$ to scenario $j$ :

$$
\mathbf{x}=\text { deaths }_{i}-\text { deaths }_{j}
$$

We can then use confidence intervals to determine the likelihood of a certain difference in outcomes between the two scenarios. For example, we may be $95 \%$ confident that the values of $\mathbf{x}$-the difference in deaths from 
scenario $i$ to scenario $j$-lie in the range $[\ell, u]$. If this range does not contain zero, i.e., the range is totally positive or totally negative, then the two scenarios are different with $95 \%$ confidence. If the range is strictly positive, then scenario $i$ is worse (more deaths) than scenario $j$ with $95 \%$ confidence; if the range is strictly negative, then scenario $i$ is better (fewer deaths) than scenario $j$ with $95 \%$ confidence.

For a desired confidence level of $(1-)$, the confidence interval $\mathrm{CI}$ is defined by

$$
\mathrm{CI}=\hat{\mathbf{x}} \pm z / 2 \hat{\mathbf{x}}
$$

where $\hat{\mathbf{x}}$ is the mean of $\mathbf{x}$ and $\hat{\mathbf{x}}$ is the standard deviation of $\mathbf{x}$. The value $z / 2$ follows from the normal distribution function.

Worker absenteeism was found to be relatively low, but this was predominately due to the assumption that workers would be outfitted with personal protective equipment. To outfit the 14,330 clinicians found in the model would cost approximately $\$ 1.15 \mathrm{M}$ a day, or $\$ 115 \mathrm{M}$ throughout the 100 -day model. Note however, that because of the shortage of and the high turnover of adequately trained emergency nurses in Ontario (Drummond 2002), further increasing worker absenteeism may again have drastic effects on the health system.

\section{CONCLUSIONS AND FUTURE DIRECTIONS}

The ability of the population to seek medical attention bears a significant impact on the resulting disease spread in a pandemic. Omission of a health system in agent-based simulation models results in a drastic underestimation of disease spread where medical facilities are readily available to the population. With a health system in place, increasing levels of medical facilities provided reduction in disease spread. Specifically, the availability of flu assessment, treatment and referral centers reduces the load on hospitals and the number of deaths in the general population. Because infected individuals now seek care at medical facilities (in this model, hospitals or primary care physicians), there is increased exposure among clinicians. However, this increased exposure is mitigated by the fact that we assume clinicians are properly outfitted with appropriate personal protective equipment, so the actual number of infections among clinicians remains low.

Future directions include incorporation of per-day assessments on hospital load, and whether or not there are indicators among census tracts or LHIN regions as to when hospitals should consider increasing their workforce, or begin preparing for significant worker absenteeism. Emergency department demand, ward bed demand, intensive care unit demand, and worker absenteeism could all be assessed and compared to available capacity on a per-hospital basis.

Although this research illustrated the usefulness of flu centers, we did not address the exact number or location of these flu centers, instead choosing to focus on the probability that an individuals will visit a flu center. Future work into optimally locating these facilities based on predictions of regional disease spread would be helpful in assisting policy-makers plan for a pandemic outbreak.

\section{ACKNOWLEDGMENTS}

This work was supported by the Leaders of Tomorrow Fund of The Canadian Foundation for Innovation.

\section{REFERENCES}

Aleman, D. M., T. G. Wibisono, and B. Schwartz. 2009a, December. Accounting for individual behaviors in a pandemic disease spread model. Proceedings of the Winter Simulation Annual Conference.

Aleman, D. M., T. G. Wibisono, and B. Schwartz. 2009b, June. A non-homogenous agent-based simulation approach to modeling the spread of disease in a pandemic outbreak. Proceedings of the IERC Annual Conference.

Centers for Disease Control and Prevention 2005. FluSurge. http://www. cdc.gov/.

Central East LHIN 2009. Integrated health service plan 2010-2013. http: //www. centraleastlhin.on.ca/.

Central LHIN 2008. 2007-2008 annual report. http: / /www. centrallhin. on. ca/.

Central West LHIN 2004. Population health profile: Central West. http: //www. centralwestlhin.on.ca/.

Drummond, A. J. 2002. No room at the inn: overcrowding in Ontario's emergency departments. Canadian Journal of Emergency Medicine 4 (2): 91-97.

Institute for Clinical Evaluative Sciences 2000. Emergency department services in Ontario. http://www.ices.on.ca/. 
Larson, R. 2007. Simple models of influenza progression within a heterogeneous population. Operations Research 55 (3): 399-412.

Low, D., K. Bartlett, J. Baudouin, A. Bourgault, L. Brosseau, P. Ericson, M. Gardam, R. Janssen, A. McGeer, L. O'Brian-Pallas, G. Stiver, S. Tamblyn, and R. Tellier. 2007. Influenza transmission and the role of personal protective respiratory equipment: An assessment of the evidence. Report in Focus by the Council of Canadian Acedemies.

Meyers, L., B. Pourbohloul, M. Newman, D. Skowronski, and R. Brunham. 2005. Network theory and SARS: Predicting outbreak diversity. Journal of Theoretical Biology 232 (1): 71-81.

Mississauga Halton LHIN 2004. Population health profile: Mississauga Halton. http://www. mississaugahaltonlhin.on.ca/.

National Physician Survey 2007. National physician survey. http: / / www . national physiciansurvey . ca/.

Newman, M. E. J. 2002. Spread of epidemic disease on networks. Phys. Rev. E 66 (1): 016128.

Ontario Health Plan for an Influenza Pandemic 2008. Ontario health plan for an influenza pandemic. http: //www.health.gov.on.ca/.

Ontario Hospital Association 2007. Hospital report. http: / /www . oha. com/.

Ontario Physician Human Resources Data Centre 2008. Annual Report: Physicians in Ontario. https: //www.ophrdc.org/.

Toronto Academic Health Sciences Network 2006. Pandemic influenza planning guidelines. http://saturn.pointalliance.com/.

Toronto Central LHIN 2004. Population health profile: Toronto Central. http://www.torontocentrallhin.on.ca/.

Valle, S. D., J. Hyman, H. Hethcote, and S. Eubank. 2007. Mixing patterns between age groups in social networks. Social Networks 29:539-534.

\section{AUTHOR BIOGRAPHIES}

NATALIA E. LIZON Natalia Lizon is a recent baccalaureate graduate of Industrial Engineering from the University of Toronto. Her professional interests include the application of system-level thinking and industrial engineering tools in healthcare settings. She has previously developed a cost/benefit model for the standardization and automation of health IT for the province of Ontario. She has also engaged over 20 acute and community organizations in the planning for an electronic health record for the Greater Toronto Area. Ms. Lizon has previously consulted with Courtyard Group, worked with the Ministry of Health and Long-Term Care, and consulted independently for the Centre for Addiction and Mental Health. Ms. Lizon's email address is<nat.lizon@utoronto.ca>.

DIONNE M. ALEMAN is an Assistant Professor in the Department of Mechanical and Industrial Engineering at the University of Toronto. She received her Ph.D. in Industrial and Systems Engineering from the University of Florida in Gainesville, FL. Her research interests are medical applications of operations research, specifically, pandemic outbreak planning and radiotherapy treatment optimization. She serves as the Chair of the INFORMS Health Applications Section, the Treasurer of the INFORMS Junior Faculty Interest Group and is the director of the Medical Operations Research Lab (morLAB) at the University of Toronto. Professor Aleman's email address is <aleman@mie.utoronto.ca>.

BRIAN SCHWARTZ is the Director of the Emergency Management Support of the Ontario Agency for Health Protection and Promotion (OAHPP), and Scientific Advisor for the Emergency Management Unit, Ontario Ministry of Health and Long Term Care. He has collaborated on health services and systems research in emergency medical dispatch, emergency department overcrowding and mitigation strategies for pandemic influenza, and has supervised Master's and Doctorate level students in faculties of Medicine and Engineering. Dr. Schwartz is also an Associate Professor in the Department of Family and Community Medicine at the University of Toronto, and during the recent 2009 Pandemic H1N1 was Chair of the Ontario Scientific Response Team. Dr. Schwartz's email address is <brian.schwartz@oahpp.ca>. 